\title{
Microesferocitosis Familiar Estudio en 10 Familias
}

\author{
Dra. Ana Becker C. ${ }^{1}$; Dra. M. Angela Delucciti B. ${ }^{1}$; Dra. Yamil Hodali $\mathrm{H}^{2}$; \\ Dr. Hernán Taboada L. ${ }^{3}$; T.M. Nereyda Valiente $Z^{9}$ \\ Hereditary Spherocytosis \\ A study of ten families
}

A $61 / 2$ years old girl admitted to our hospital with an aplastic crisis, a serious complication of Hereditary Spherocytosis (H.S.), motivated a retrospective review of the cases studies in our Hematology Department during the last seven years. Ten families were studied finding 20 members with H.S. The diagnosis was made in most of them during school age and only in two patients during the neonatal period. Most patients ( $80 \%$ ) bad objective family history of H.S. Hemolytic crisis represented $80 \%$ aplastic crisis $15 \%$ and chojelithiasis $5 \%$ of the complications detected among our patients. The relationships that exist between aplastic crisis and viral infections, specially parvo-virus, is stressed. It is important to point out that two out of three mothers who were not treated by splenectomy, showed severe hemolitic crisis during their pregnancy. Splenectomy, its indications and posterior anti-infective prophylaxis are discussed.

(Key words: Anemia. Hemoly sis. Hereditary Spherocy tosis. Aplastic crisis. Splenectomy).

A propósito de la admisión en nuestro servicio de una escolar con microesferocitosis familiar (MF), cuyo diagnóstico se hizo a los 6 años a raíz de una crisis aplástica, tuvimos un problema diagnóstico pues no existían antecedentes familiares. Esto ros impulsó a revișar nuestra experiencia y otras publicaciones referentes a los sintomas de presentación de la enfermedad, sus complicaciones, tratamiento y evolución pre $y$ post esplenectomía.

\section{MATERIAL Y METODOS}

Desde 1978 hasta la fecha hemos controlado 10 familias con MF, de cuyos miembros fueron registrados la edad en el momento del diagnóstico: los síntomas de presentación; antecedentes perinatales y familiares; evolución de la enfermedad; complicaciones de ésta $y$ trata-

1. Médico pediatra. Servicio de Pediatría. Hospital Sótero del Río. S.S.M.S.O.

2. Becario Pediatría, Universidad Católica de Chile. Hospital Sótero del Río. S.S.M.S.O.

3. Jefe Hematología, Servicio de Pediatría. Hospjtal Sótero del Río. S.S.M.S.O.

4. T. Médica, Servicio Hematología, Hospital Sótero del R io. S.M.M.O. miento. En todos los casos se realizaron hemogramas con recuentos de reticulocitos, pruebas de fragilidad osmótica, pruebas de Coombs, pruebas hepáticas y últimamente, antes de la esple. nectomía, ecografía vesicular en busca de colelitiasis.

\section{RESULTADOS}

Nuestros 10 casos indices (Tabla 1 ) nos condujeron a 20 pacientes, 10 hombres y 10 mujeres con MF. Sólo en 2 casos el diagnóstico se realizó en el período de recién nacido $(\mathrm{RN})$, en la mayoría se hizo en la época escolar (entre 5 y 12 años), y en una proporción no desprecia. ble después de los 18 años de edad. Cabe destacar que en dos madres la enfermedad fue reçonocida en el embarazo a raíz de crisis hemolíticas que les produjeron anemia severa.

En el 70\% de nuestros casos índices el motivo de consulta fue anemia de causa no identificada. En 2 pacientes en que el diagnóstico se fonmuló en el período de $\mathrm{RN}$, las madres tenían anemia severa en el tercer trimestre del embarazo y estaban siendo estudiadas en búsqueda de microesferocitosis.

Los demás pacientes no consultaron en forma espontánea siendo detectados durante el ras- 
Tabla I

Microesferocitosis Familiar

Casos - Indices

$1978-1985$

\begin{tabular}{|c|c|c|c|c|}
\hline Familia & $\begin{array}{c}\text { Edad al Diag. } \\
\text { (Años) }\end{array}$ & $\begin{array}{l}\text { Motivo de } \\
\text { Consulta }\end{array}$ & $\begin{array}{l}\text { Antecedentes } \\
\text { Perinatales }\end{array}$ & $\begin{array}{l}\text { Antecedentes } \\
\text { Familiares }\end{array}$ \\
\hline 1 & R.N. & $\begin{array}{l}\text { Hiperbilirubinemia } \\
\text { Madre c/anemia }\end{array}$ & $\begin{array}{c}\text { Ictericia } \\
y \\
\text { Exanguíneotransf. }\end{array}$ & + \\
\hline Il & R.N. & $\begin{array}{l}\text { Hiperbilirubinemia } \\
\text { Madre c/anemia }\end{array}$ & $\begin{array}{c}\text { Ictericia } \\
y \\
\text { Exanguineotransf. }\end{array}$ & + \\
\hline III & $01 / 12$ & $\begin{array}{c}\text { Anemia Severg } \\
\text { C.N.P.* }\end{array}$ & $\begin{array}{c}\text { Ictericia } \\
+ \\
\text { Fototerapia }\end{array}$ & + \\
\hline rV & $30 / 12$ & $\begin{array}{l}\text { Heilazgo al } \\
\text { Hernograma }\end{array}$ & - & + \\
\hline $\mathrm{v}$ & $36 / 12$ & $\begin{array}{l}\text { Anemia } \\
\text { C.N.P.* }\end{array}$ & - & + \\
\hline VI & $58 / 12$ & $\begin{array}{l}\text { Anemis } \\
\text { C.N.P." } \\
\text {. }\end{array}$ & $\begin{array}{c}\text { Ictericia } \\
+ \\
\text { Fototerapin }\end{array}$ & + \\
\hline VII & $66 / 12$ & $\begin{array}{l}\text { Anemia Severa } \\
\text { Arregenerativa }\end{array}$ & Ictericia & - \\
\hline VIII & $84 / 12$ & $\begin{array}{l}\text { Anemia } \\
\text { C.N.P." }\end{array}$ & - & + \\
\hline IX & $100 / 12$ & $\begin{array}{l}\text { Anemia } \\
\text { C.N.P.* }\end{array}$ & - & - \\
\hline $\mathbf{x}$ & $102 / 12$ & $\begin{array}{l}\text { Anemia } \\
\text { C.NP.* }\end{array}$ & - & + \\
\hline
\end{tabular}

* C.N.P. $=$ Causa no precisada.

treo de familiares afectados de los casos indices.

Existían antecedentes familiares sugerentes de MF en $80 \%$ de los pacientes indices. De las 10 familias estudiadas, 7 tenían antecedentes maternos y en sólo una la enfermedad habia sido transmitida presumiblemente por el padre. Todas las madres fueron esplenectomizadas, pero en dos la intervención se realizó después de haber sufrido crisis hemolítica severa en el curso de sus embarazos.

Cinco de los casos índices tuvieron ictericia en el período de $\mathbf{R N}$; dos debieron ser tratados con exanguíneotransfusiones y, sólo en éstos, el diagnóstico fue hecho oportunamente. Al res- pecto cabe destacar que en uno de los hermanos de un caso indice, se realizó exanguineotransfusión en el período de $R N$ por ictericia $y$, a pesar de tener antecedentes familiares anpliamente positivos, no se hizo diagnóstico correcto sino hasta los 9 años de edad.

El hematocrito promedio, en los 20 pacientes estudiados era de $29 \%$ (máx. 35\% Mín. 21\%) y el recuento promedio de reticulocitos 8,5\% (máx. 12\% min. 5\%) durante su evolución habitual, antes de la esplenectomía. En todos sus frotis de sangre periférica había microesferocitosis y la fragilidad osmótica de los glóbulos rojos (GR) estaba aumentada, con respecto a 
los normales, al someterlos a concentraciones decrecientes de $\mathrm{NaCl}$. Las pruebas de Coombs eran negativas $y$ las transaminasas normales en todos los casos.

La exploración ecográfica de la vesícula se realizó en 5 de 12 pacientes antes de la esplenectomía y no mostró cálculos. Una paciente de 28 atos fue intervenida quirúrgicamente de urgencia, por colelitiasis, practicándosele esplenectomia simultáneamente.

En 12/20 pacientes ocurrieron 20 episodios de complicaciones. (Tabla 2).

Tabln 2

Microesferocitosis Familiar

Estudio en 10 Familins

\begin{tabular}{lcc}
$\begin{array}{l}\text { Total de Pacientes } \\
\text { Total de Pacientes c/complicaciones }\end{array}$ & $\begin{array}{l}= \\
= \\
=\end{array}$ \\
\hline $\begin{array}{l}\text { Tipos de Complicaciones } \\
\text { No Obsv. }\end{array}$ & $\%$ \\
\hline $\begin{array}{l}\text { Hiperbilirubinemias en R.N. } \\
\text { Crisis Hemolíticas }\end{array}$ & 8 & 40 \\
Crisis Hemolíticas 3er. Trim. & 6 & 30 \\
$\quad$ Embarazo & 2 & 10 \\
Crisis Aplásticas & 3 & 15 \\
Coleititiasis & 1 & 5 \\
\hline Total de Complicaciones & 20 & 100 \\
\hline
\end{tabular}

La complicación más frecuente fue la crisis hemolítica; las hiperbilimubinemias en el período de $\mathrm{RN}$ se consideraron parte de éstas y se presentaron en 8 pacientes, de los que 5 requirieron fototerapia y 3 exanguíneotransfusiones. Las crisis hemoliticas posteriores al período de RN se observaron en 8 oportunidades, 2 durante el 3er. trimestre de un embarazo. Por lo tanto las crisis hemolíticas constituyeron el $80 \%$ de las complicaciones observadas. En tres cuartos de ellas el hematocrito fue inferior a $24 \%$, con recuentos promedios de reticulosis mayores que $17 \%$. En un paciente el hematocrito disminuyó hasta $13 \%$ durante la crisis, que en más de la mitad de los casos ocurrió en coincidencia con sintomas sugerentes de infecciones.

Las crisis aplásticas correspondieron a $15 \%$ de las complicaciones; todos los pacientes ingresaron al hospital con anemia severa, con Hicto. de $13 \%$ en promedio (máx. $18 \%$ min. 10\%) y reticulocitos promedio de $0,6 \%$ (máx. 1,7\% y min. $0 \%$ ). La duración de la crisis fue aproximadamente de 2 semanas recuperándose en forma satisfactoria, con reaparición de reticulocitos. En todos los casos fue necesario emplear transfusiones.

Una pacjente que presentó colelitiasis a la edad de 28 afios ya fue descrita en párrafos anteriores.

Todos los pacientes fueron tratados con suplementos de ácido fólico antes de la esplenec. tomía. En los casos que tenían anemia severa, secundaria a crisis hemolítica o aplástica, se indicaron transfusiones de glóbulos rojos como ya se señaló. En 11 pacientes se realizó esplenectomia entre las edades de 8 y 34 años.

Después de la esplenectomía se usó profilaxis con Penicilina Benzatina en 4 pacjentes, los cuales viven alejados de un centro hospitalario. E1 resto recibió indicaciones precisas para acudir a un centro asistencial en caso de fiebre y en los pacientes más recientes se han inyectado vacunas neumocócicas.

La madre de uno de nuestros casos indices falleció 2 años después de la esplenectomía, debido a menjngitis purulenta de la que no se aisló el agente causal. La paciente no estaba tecibiendo profilaxis.

\section{DISCUSION}

La microesferocitosis familiar (MF) es la anemiạ hemolítica hereditaria más frecuente en pediatría y tiene una incidencia de 2 a $4 \times 10.000$ habitantes ${ }^{1,2}$. En $75 \%$ de los casos ia enfermedad se hereda por un patrón autosómico dominante. Existen evidencias que sugieren que el gen trasmisor se encontraría en el cromosoma $8^{2}, 3,4$. El defecto molecular está ubicado en el esquele to proteico de la membrana del eritrocito. Este citoesqueleto esta constituido fundamentalmente por espectrina, cuya molécula tiene 2 cadenas $(\alpha$ y $\beta)$ que se unen para formar un tetrámero. Los extremos libres se unen a su vez en la $\mathrm{F}$ - actina por medio de la proteina 4.1 para formar así una cadena o malla interconectada. Otras 2 proteinas -ankyrina y proteína 3- unen cada unidad de esta cadena a la capa lipídica exterior (Figura 1). En ratas se ha observado que el déficit de espectrina produce esferocitosis y hemólisis intensa. Estudios en la especie humana han permitido comprobar por lo menos tres tipos de defectos en la esferocitosis hereditaria.

El déficit de espectrina (MF [Esp +]) se caracteriza por $40 \%$ de escasez de cadena $\alpha$ y $\boldsymbol{\sigma}$, es de herencja autosómica recesiva, mayor severidad y respuesta incompleta a la esplenectomía. Es la forma más tara (1 en 35 familias estudiadas). 


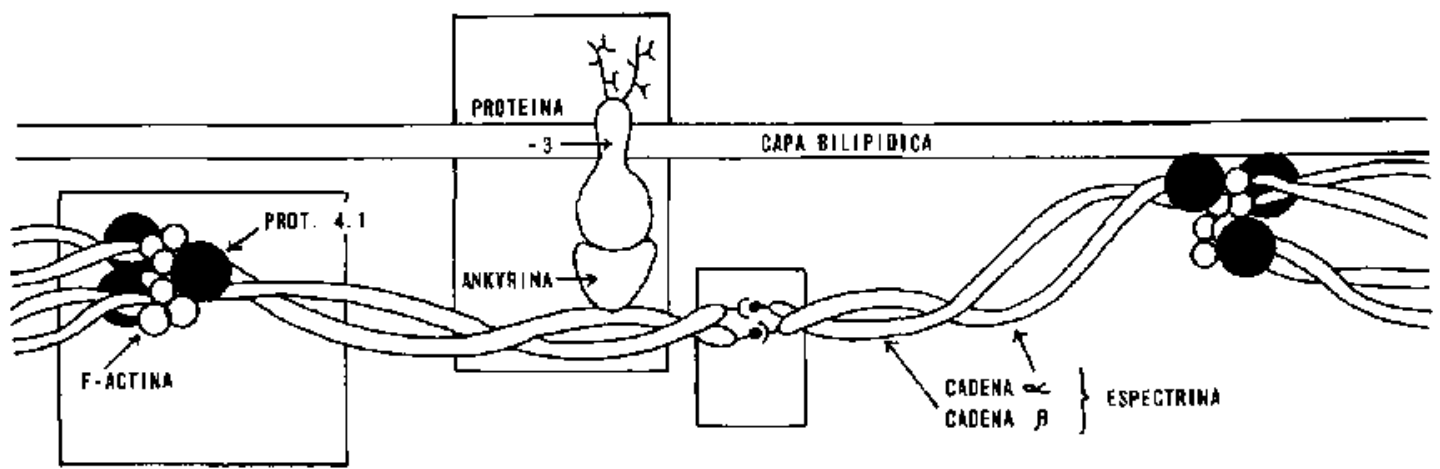

Figura 1: Interacciones de las proteínas de membrana del eritrocito.

La unión defectuosa de espectrina a la proteina 4.I (MF [Esp-4.1]) es clínicamente idéntica a las formas más frecuentes de $M F$, salvo un caso que tenia, además, aproximadamente $10 \%$ de acantocitos. El defecto afecta en $40 \%$ a la unión de las dos proteínas involucradas, lo que está de acuerdo con un estado heteroci. gótico de la herencia autosómica dominante. Su frecuencia fue 4 en 11 familias estudiadas.

Otros defectos no bien caracterizados afectarian la unión de la espectrina con la capa lipídica exterior, to que podría involucrar una unión incompleta de espectrina-ankyrina, o de la proteína 4.1 y la capa exterior, por medio de la glicoforina $C$. Se postula también que pueda ocurrir fosforilación defectuosa de la espectrina debido a una proteinoquinasa alterada o por un defecto en el carbono terminal de la cadena $\beta$, lo que comprometeria la unión dímero-dímero para constituir el tretrạmero de espectrina. Esta - estas alteraciones se encontrarían en la mayoría de los casos de MF (23 de 24 estudiados), incluso en algunas de la segunda categoría descrita.

Todas las formas tienen en común el aumen. to de la desintegración de glóbulos rojos frente a concentraciones bajas de urea, lo que podria reflejar diferencias de sensibilidad frente a oxidantes. Los eritrocitos son más permeables al $\mathrm{Na}^{+}$, lo que se traduce en aumentos de la activjdad metabólica para elevar la tasa de ATP y activar la "bomba de Sodio".

Todo lo anterior, se traduce en unas altera. ciones de la forma, flexibilidad e integridad estructural de los glóbulos rojos, disposición e inestabilidad de la capa lipídica y fusión de su membrana 5 .

Los signos y síntomas de la MF varían con la edad y severidad de la hemólisis, to que explica que el diagnóstico sea a veces tardío.
Entre sus complicaciones se describen crisis hemolíticas, aplásticas, megaloblásticas, colecistopatías y otras6, 7 .

Las complicaciones más frecuentes de la MF son las crisis hemolíticas, que se caracterizan por aumento de la anemia, reticulocitosis y esplenomegalia de grado varjable ${ }^{7}$. Generalmente ocurren en concomitancia con infecciones virales febriles y raras veces son severas.

Las crisis aplásticas son, clínicamente menos frecuentes pero más severas y se acompañan de anemia importante $\mathrm{e}^{2,7}$. En ellas el hematocrito disminuye, lo mismo que el recuento reticulocitario. Al disminuir las células nuevas, los esferocitos se hacen más evidentes en el frotis y la fragilidad osmótica aumenta. Desde hace tiempo se ha pensado que ellas guardan relación con infecciones $y$, si bien pueden ocurrir en asociación con algunas producidas por organismos específicos, como estreptococos neumoniae y microplasma neumoniae, en la mayoria de los casos no se ha logrado aislar un agente infeccioso, generalmente afectan a niños menores de 16 afros y suelen ser precedidas por sintomas sugerentes de infecciones virales. Se ha pensado que la crisis aplástica sea una respuesta no especifica a la infección, pero el hecho que los pacientes rara vez tengan más de un episodio, hace probable que pueda tratarse de un solo agente etiológicos. Existen varios indicios de que este agente etioló. gico podria ser el Parvo Virus $B_{1}$, (Human Parvo Virus -H.P.V.) ${ }^{8}, 9,10$. Las infecciones por parvo virus son comunes presentándose más frecuentemente en niflos y pueden estar asociadas a cefalea, fiebre, malestar general y erupción cutánea (exantema infeccioso o quinta enfermedad). El mecanismo de transmisión del agente aún es desconocido y la mayoría de los niños adquieren anticuerpos entre los 4 y 6 antos. Los porladores de anemia hemolítica no son más 
susceptibles que los normales a las infecciones por H.P.V. y no todos los niños que se infectan con él tienen crisis aplásticas. Una đetenciốn temporal de la eritropoyesis es común a todas las infecciones por parvo virus, pero las crisis apläs. ticas no se detectarian en individuos normales debido a la mayor sobrevida de sus glóbulos rojos y la naturaleza autolimitada de la aplasia.

Las crisis aplásticas pueden encontrarse en coalquier condición asociada a disminución de la vida media de los glóbulos rojos; es así como se han descrito, asociadas a infecciones por H.P.V., en microesferocitosis familiar, déficit de piru. vato-kinasa, $\beta$ talasemia, anemia de células falciformes y otras ${ }^{8}, 9,10$.

Las crisis aplásticas constituyen riesgo serio para los pacientes y estos deben hospitalizarse para observarlos cuidadosamente y darles tratamiento oportuno, ya que sus hematocritos pueden disminuir tanto como hasta $10 \%$ como ocurrió en uno de nuestros pacientes. Debido a que otros miembros de la familia se encuentran en riesgo de hacer esta crisis, ellos deberán ser vigilados de cerca para pesquisar trastornos similares.

En una de las familias de este estudio, los hermanos de un paciente hospitalizado por crisis aplástica presentaron simultaneamente crisis hemolítica, que en uno de ellos obligó a hospitalizarlo; ambos casos podrian ser interpretados como aspectos de la recuperación de una crisis aplástica ${ }^{9}$.

El tratamiento de la MF se ha dividido en 3 fases: Pre-esplenectomía, Esplenectomia y Post-esplenectomia ${ }^{2}$. En el período de preesplenectomía, ocasionalmente son necesarias medidas de soporte frente a episodios hemoliticos significativos. Así, los RN con hiperbilirrubinemia severa, están en riesgo de hacer kernicterus y deben ser tratados con fototerapia o exsangúneotransfusión. En algunos casos la anemia puede ser suficientemente severa como para causar sintomas, debienda emplearse transfusiones de glóbulos rojos, especialmente durante las crisis hemolíticas o aplásticas. Para prevenir las crisis megaloblásticas secundarias a la escasez de ácido fólico que se produce por hiperplasia medular, se recomienda aportar ácido fólico en dosis de $1 \mathrm{mg}$ diario. Los depósitos de vitamina $\mathbf{B}_{12}$ son adecuados en los niños y no es necesario emplear suplemento de ella ${ }^{1}$. Tampoco es necesario administrar fietro después del primer afio de vida, ya que este es vuelto a usar en la formación de hemoglobina ${ }^{6,7}$. Incluso en las anemias hemolíticas crónicas pueden ocurrir sobrecargas de Fe por aumentos de la absorción.

La esplenectomia reduce la hemólisis y alivia la anemia, permitiendo a los eritrocitos circulan- tes sobrevivir períodos cercanos a los del glóbulo rojo normal. Por ello las crisis aplásticas dejan de ser un problema significativo $2,3,6,7$. La colelitiasis es consecuencia de la hemólisis crónica y también se reduce con el procedimiento, por esta razón la esplenectomía se emplea como tratamiento de la $\mathrm{MF}^{2}, 3,6,7$. Sin embargó, el bazo tiene una función importantísima en la protección contra las infecciones, lo que obliga a ser más cauteloso con el empleo de la esplenec. tomia de rutina en niños que sufren de la enfermedad.

Balanceando riesgos y beneficios, se recomienda realizar esplenectomía en todos los pacientes que sufran anemia, hemólisis significativa (recuentos de reticulocitos mayores a $5 \%$ ) e historia familiar de colelitiasis ${ }^{7}$. Nosotros creemos que la esplenectomía debe ser propuesta en las mujeres con MF que pretenden procrear por el alto riesgo de crisis hemolíticas durante el embarazo; dos de las tres madres no esplenecto. mizadas de nuestra casuística, tuvieron crisis hemolíticas severas en el tercer trimestre del embarazo 11, 12 .

Debido al mayor riesgo de septicemia que existe para los lactantes y preescolares se recomienda aplazar la esplenectomia hasta los 6 años No existen evidencias respecto a que postergaciones mayores sean de utilidad, pero en cambio el riesgo de cálculos de la vía biliar aumenta notablemente despúés de los 10 años ${ }^{7}$. Antes de realizar la esplenectomia se recomienda la inmunización con vacuna neumocócica en todos los nifios ${ }^{2}, 7$.

Después de la esplenectomía, los pacientes están sometidos a riesgo de infecciones por microorganismos encapsulados, especialmente estreptococo pneumoniae y hemofilus influenzae El riesgo es mayor en los pacientes esplenectomizados antes de los 2 años, pero siempre es alto en cualquier edad en comparación con niños normales ${ }^{7}, 13$. Según un informe nacional ${ }^{13}$, no ocurrieron complicaciones en pacientes esplenectomizados por MF, seguidos en promedio por 3 años; en nuestra casuistica su riesgo de sufrir infecciones graves $y$ muerte fue de $2,3 \%$, como en otras experjencias ${ }^{14}$. En otras series de niños esplenectomjzados por traumatismos esplénicos, la morbilidad fue de $1,5 \%$ con $40 \%$ de morbilidad por infección 7 , la que podria disminuir con inmunizaciones con vacuna neumo. cócica pues $50 \%$ de las septicemias en pacientes con esplenectomía son causadas por estreptococo neumoniae y $80 \%$ de estas son producidas por alguna de las 14 cepas cuyos polisacáridos incluyen las vacunas habituales ${ }^{15}$; cuyo contenido se ha ampliado recientemente a las de un total de 23 cepas $^{15}$. No existe una conducta uniforme res- 
pecto al uso profiláctico de antibióticos, y no será fácil lograrlo pues han aparecido cepas de neumococos resistentes a la penicilina $y$, por otra parte el otro $50 \%$ de las septicemias es producido en proporciones similares por Neisseria meningitis, Escherichia coli, Hemofilus influenzae, Estafilococus aureus y Estreptococus sp., todos con diferentes patrones de sensibilidad a los antimicrobianos.

Existe, sin embargo, acuerdo en que el niño esplenectomizado antes de los 6 años debe recibir antibióticos como profilaxis; no ocurre lo mismo respecto al nińo mayor y el adulto.

El antibacteriano de elección es la penicilina, pero existe una tendencia creciente a usar drogas capaces de controlar el $\mathrm{H}$. influenzae. No hay datos definitiyos en apoyo de una $u$ otra conduc. ta; sin embargo las drogas diferentes de la penicilina son caras, $y$ no hay experiencia sobre sus efectos en la flora intestinal y atros secundarios cuando se las emplea por largo tiempo.

Se sugiere el uso profiláctico de penicilina oral en dosis de. 125 a $250 \mathrm{mg}$., 2 veces por día en pacientes de 2 a 6 años. Después de $\operatorname{los} 6$ años, algunos recomiendan usar amoxicilina oral cuando aparece fjebre y consulta inmediata ${ }^{6}$.

En los pacientes esplenectomizados que vuelven a lugares de origen alejados de los centros asistenciales se recomienda profilaxis con Penicilina Benzatina cada 28 días en los primeros 2 anos que siguen a la esplenectomía, que son los de mayor riesgo ${ }^{13}$. En los niños que tienen fácil acceso a los centros asistenciales sugermos controles médicos periodicos reforzando a través de ellos la conducta más adecuada frente a cuadros febriles.

\section{RESUMEN}

A taíz de 10 casos indices, se estudiaron 10 familias con un total de 20 pacientes que sufrian microesferocitosis hereditaria. El diagnóstico fue tardío, en la mayoría de los casos en edad escolar. En sólo 2 niños la enfermedad fue identificada en el periodo de $R N$ porque sus madres estaban en estudio por anemia y sufrieron crisis hemoliticas durante el embarazo. De un total de 20 episodios de complicación, las crisis hemolíticas constituyeron el $80 \%$, las crisis aplásticas el $15 \%$ y la colelitiasis el $5 \%$. Las crisis aplásticas fueron más severas porque fueron acompañadas por anemia importante.

\section{REFERENCIAS}

1. Bravo M., Vikósola J.: Anemias en el lactante menor. Bol Hosp. San Juan de Dios, 25: 239, 1980.

2. Sullivan D.W., Glader B.E.: Hereditary Spherocytosjs. Pediatr. Ann 9: 308, 1980.

3. Rencoret G., Vorgas L., Garcis H.: Esferocitosis Hereditaria. Bol, Hosp. San Juan de Dios 26: 294, 1979.

4. Bass B.E., Smith W.S. et aL: Further evidence for location of the Spherocytosis gene on cromosome 8. Ann Jnt. Med. 99: 192, 1983.

5. Polek $J$., Somuel $S . E$.: Red cell mombrane skeletal defects in hereditary and acquired hemolytic anemias. Seminars Hematol. 20: 189, 1983.

6. Figuera R., Rivera $M$ : Esferocitosis Familiar Congénita. Rev. Chil. Pedjatr. 54: 340, 1983.

7. Lux S.E. Wolfe L.C.: Inherited disorders of the red cell membrane skeleton. Pediatr. Clin North. Amer. 27: 453,1980 .

8. Editorial: Bone-marrow aplasia and Parvovirus. Lancet 2: 21, 1983

9. Kelleher J.F., Luban J.Ln, Mortimer P.P, et al. Human serum "parvovirus". A specific cause of aplastic crisis in children with hereditary spherocytosis. J. Pediatr. 102: 720, 1983.

10. Duneen J.R. y Cols: Aplastic crisis due to parvovirus infection in pyruvate kinase deficiency. Lancet 2: 14, 1983.

11. Venturo C.S.: Hereditary spherocitosis with haemolytic crisis during pregnancy. Aust N.Z.J. Obstet. Gynaecol 122: 50, 1982.

12. Ho-Yen Do: Hereditary spherocytosis presenting in pregnancy. Acta Haematol. 72: 29, 1984.

13. Brovo M. y Cols.: Esplenéctomía en niños. Revisión de 75 casos. Rev. Chil. Pediatr. 47: 203, 1976.

14. Singer B.: Postsplenectomy sepsis. Perspect. in Pediat. Pathol 1: 285, 1973.

15. Taboada H.: Vacuna Anti-neumocócica, en: Manual de Pediatría, Winter A. y Puentes R. (Ed.), Hospital Sótero del Río y Universidad Católica, Santiago de Chile, 1984, Tomo I pág. 342

16. Giebink G,S: Preventing pneumococeal disease in children. Pediatr. Infect. Dis. 4: 343, 1985. 